\title{
Search for a possible space-time correlation between high energy neutrinos and gamma-ray bursts ${ }^{\star}$
}

\author{
T. Montaruli ${ }^{1}$ and F. Ronga ${ }^{2}$ for the MACRO collaboration ${ }^{3}$ \\ 1 INFN, Dipartimento di Fisica, Università di Bari, via Amendola 173, I-70126 Bari, Italy \\ 2 INFN, Laboratori Nazionali di Frascati, P.O. Box 13, I-00044 Frascati, Italy \\ 3 For the collaboration list see (Ambrosio 1998a)
}

Received December 20, 1999; accepted January 4, 2000

\begin{abstract}
We look for space-time correlations between 2233 gamma-bursts in the Batse Catalogs and 894 upward-going muons produced by neutrino interactions in the rock below or inside MACRO. Considering a search cone of $10^{\circ}$ around GRB directions and a time window of $\pm 200 \mathrm{~s}$ we find 0 events to be compared to 0.035 expected background events due to atmospheric neutrinos. The corresponding upper limit (90\% c.l.) is $0.8710^{-9} \mathrm{~cm}^{-2}$ upward-going muons per average burst.
\end{abstract}

Key words: gamma-ray bursts (GRB)

\section{Neutrino astronomy with MACRO}

Besides high energy gamma ray production resulting from $\pi^{0}$ decay, astrophysical beam dump models predict neutrino emission from $\pi^{ \pm}$decay. Mesons are produced by accelerated protons interacting with matter or photons in an accretion disk (Gaisser 1995). The discovery of TeV gamma-ray emissions has enhanced the potential possibilities of this mechanism and the possible existence of such sources, but energies are not high enough to exclude syncroton radiation or bremsstrahlung and inverse Compton production mechanisms. Neglecting photon absorption, it is expected that neutrino fluxes are almost equal to gamma ray ones and that the spectrum has the typical form due to the Fermi acceleration mechanism: $\frac{\mathrm{d} N}{\mathrm{~d} E} \propto E^{-2.0 \div 2.2}$. Neutrinos produced in atmospheric cascades are background to the search for astrophysical neutrinos for energies $\lesssim 10 \mathrm{TeV}$. In fact, atmospheric neutrinos have a softer spectrum than astrophysical neutrinos,

Send offprint requests to: T. Montaruli

* This article had been submitted as a contribution paper in the "Rome '98 gamma-Ray Burst workshop" and should have appeared in the A\&AS special issue for the workshop (A\&AS 1999, $138(3))$. since at energies $\gtrsim 100 \mathrm{GeV}$ the decay length of mesons in the atmosphere becomes longer than atmospheric depth and the spectrum steepens (differential spectral index $\gamma \simeq$ 3.7). GRBs are possible sources of high energy $\nu$ s: in the fireball scenario the beam dump mechanism can lead to $\nu$ emission (Halzen 1996; Waxman 1997). The short time duration of the GRB emission allows to reduce the atmospheric neutrino background thanks to the additional requirement on time coincidence with the GRB emission besides the directional coincidence. Hence, although the $\nu$ expected fluxes from GRBs are much lower than the atmospheric $\nu$ flux, the time and directional association between GRBs and neutrinos can reach interesting sensitivities in underground detectors.

The MACRO detector, located in the Hall B of the Gran Sasso underground laboratories, with a surface of $76.612 \mathrm{~m}^{2}$ and a height of $9 \mathrm{~m}$, can indirectly detect neutrinos using a system of $\sim 600$ ton of liquid scintillator to measure the time of flight of particles (resolution $\sim 500 \mathrm{ps}$ ) and $\sim 20000 \mathrm{~m}^{2}$ of streamer tubes for tracking (angular resolution better than $1^{\circ}$ and pointing accuracy checked using moon shadow detection (Ambrosio 1998a). The time of flight technique allows the discrimination between downward-going atmospheric muons and upward-going events produced in the rock below (average atmospheric neutrino energy $\left\langle E_{\nu}\right\rangle \sim 100 \mathrm{GeV}$ ) and inside $\left(\left\langle E_{\nu}\right\rangle \sim 4 \mathrm{GeV}\right)$ the detector by neutrinos which have crossed the Earth. Between $\sim 3110^{6}$ atmospheric muons, a sample of 909 upward-going muons is selected with an automated analysis. The data taking has begun since March 1989 with the incomplete detector (Ahlen 1995) and since April 1994 with the full detector (Ambrosio 1998b). In our convention $1 / \beta=\Delta T c / L$, calculated from the measured time of flight $\Delta t$ and the track length between the scintillator layers, is $\sim 1$ for downward-going muons and $\sim-1$ for upward-going muons. Events with $-1.25<1 / \beta<-0.75$ are selected. 
We look for a statistically significant excess of $\nu$ events in the direction of known $\gamma$ and X-ray sources (a list of 40 selected sources, 129 sources of the $2^{\text {nd }}$ Egret Catalogue, 2233 Batse GRB, 220 SN remnants, 7 sources with $\gamma$ emission above $1 \mathrm{TeV}$ ) with respect to fluctuations of the atmospheric $\nu$ background. The expected background from atmospheric $\nu \mathrm{s}$ is calculated in declination bands of $\pm 5^{\circ}$ around the declination of the source mixing for 100 times local coordinates and times of upward-going $\mu \mathrm{s}$. We calculate flux limits in half-cones of $3^{\circ}$ taking into account reduction factors due to the signal fraction lost outside the cone (which depends on $\nu$ spectrum, kinematics of CC interaction, $\mu$ propagation in the rock, MACRO angular resolution). We do not find any signal evidence from known sources or of clustering of events (we measure 89 clusters of $\geq 3$ events and expect 81.2 of them in a cone of $3^{\circ}$ ). Muon flux limits for some sources are: 2.5 for Crab Nebula, 5.6 for MRK 421, 3.71 for Her X-1, 0.45 for Vela Pulsar, 0.65 for SN 1006 in units of $\times 10^{-14} \mathrm{~cm}^{-2} \mathrm{~s}^{-1}$. For most of the considered sources MACRO gives the best flux limits compared to other underground experiments.

\section{Space-time correlations between GRBs and upward-going muons}

We look for correlations with 2233 GRBs in the Batse Catalogs 3B and 4B (Meegan 1997) collected since 21 Apr. 1991 to 5 Oct. 1998 and 894 of the 909 upwardgoing muons detected by MACRO during this period (see Fig. 1). Considering Batse angular accuracy, we estimate that a half-cone of $20^{\circ}\left(10^{\circ}\right)$ contains $99.8 \%$ $(96.8 \%)$ of $\nu$ sources (if GRB sources are $\nu$ sources, too). The same percentages of upward-going muons are contained in these half-cones from the GRB sources. As a matter of fact, we calculate via Monte Carlo the fraction of signal lost, which depends on the $\nu$ spectral index, multiple scattering of muons during propagation in the rock and MACRO angular resolution. Using various cone apertures, we estimate that this fraction is negligible for $\geq 10^{\circ}$.

The area for upgoing muon detection in the direction of the GRBs averaged over all the bursts is $119 \mathrm{~m}^{2}$. Its value is small because MACRO is sensitive to neutrinos only in the lower hemisphere and because it was incomplete in the period 1991-1994. We find no statistically significant correlation between neutrino event and GRB directions and detection times. As shown in Fig. 2, we find no events in a window of $\pm 200 \mathrm{~s}$ inside $10^{\circ}$ from GRB directions and 1 event inside $20^{\circ}$, which was measured after $39.4 \mathrm{~s}$ from the Batse GRB of 22 Sep. 1995 (4B 950922). For this burst the radius of the positional error box in the Batse catalog is $3.86^{\circ}$, much smaller than the angular distance of $17.6^{\circ}$ at which we find the neutrino event. The expected number of atmospheric $\nu$ events is computed with the delayed coincidence technique. We

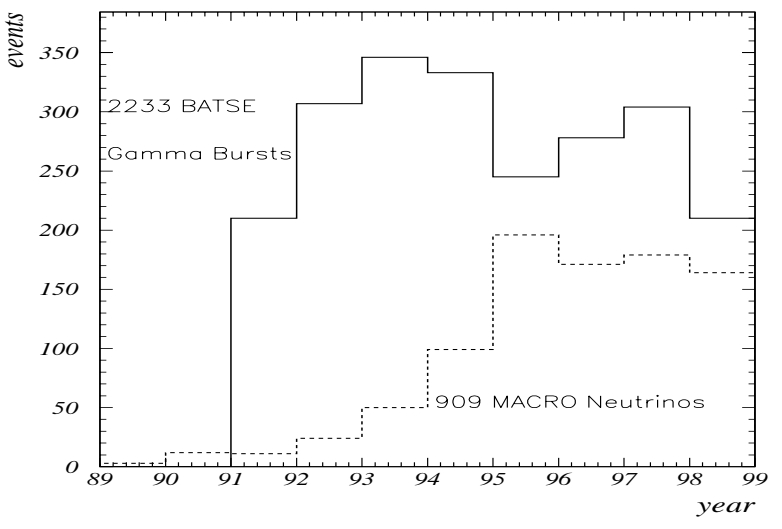

Fig. 1. MACRO upward-going muon (dashed line) and Batse GRB (solid line) distributions versus year
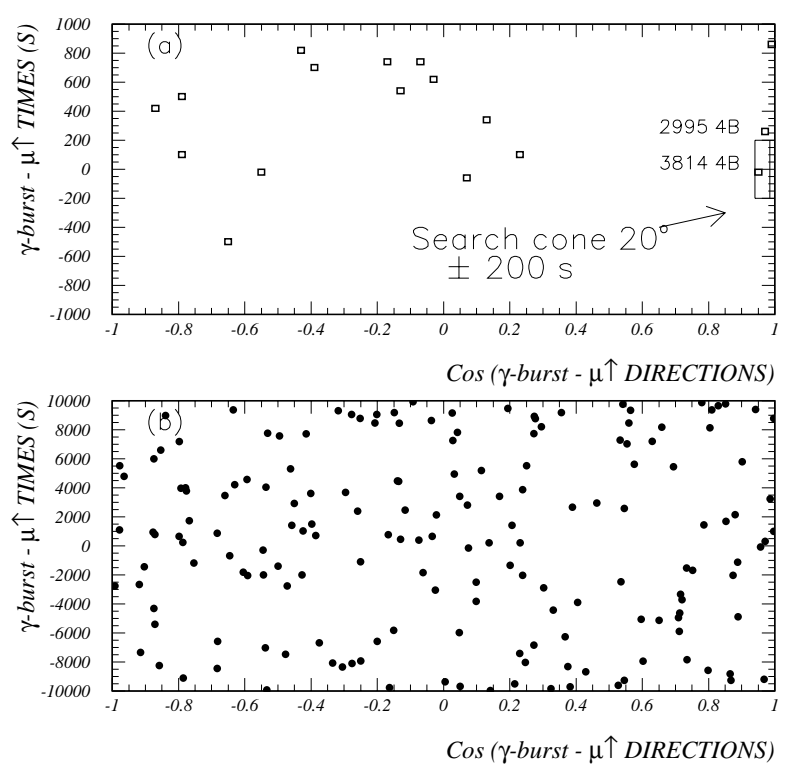

Fig. 2. Difference in detection times vs. cosine of angular separation between Batse GRBs and upward-going $\mu \mathrm{s}$. a) and b) have different time scales. In a) the $\pm 200 \mathrm{~s}-10^{\circ}, 20^{\circ}$ windows are indicated

expect $0.035(0.075)$ events in $10^{\circ}\left(20^{\circ}\right)$. The corresponding upper limits $(90 \%$ c.l.) for the upward-going muon flux are $0.8710^{-9} \mathrm{~cm}^{-2}\left(1.4410^{-9} \mathrm{~cm}^{-2}\right)$ upward-going muons per average burst. These limits exclude an extreme cosmic string-type model reported in (Halzen 1996), which results in $10^{-1} \mu \mathrm{cm}^{-2}$, while according to a fireball scenario model in (Waxman 1997), a burst at a distance of $100 \mathrm{Mpc}$ producing $0.410^{51} \mathrm{erg}$ in neutrinos of around $10^{14} \mathrm{eV}$ would produce $\sim 610^{-11} \mathrm{~cm}^{-2}$ upward-going muons. 


\section{References}

Ambrosio M., et al., MACRO Coll., 1998, Observation of the shadowing of cosmic rays by the Moon using a deep underground detector, hep-ex/9807006, accepted by Phys. Rev. D

Ahlen S., et al., MACRO Coll., 1995, Phys. Lett. B357, 481
Ambrosio M., et al., MACRO Coll., 1998, Phys. Lett. B434, 451

Gaisser T.K., et al., 1995, Phys. Rep. 258, 173

Halzen F., Jaczko G., 1996, Phys. Rev. D 54, 2779

Meegan C.A., 1997, Current Batse Gamma-Ray Burst Catalog, http://www.batse.msfc.nasa.gov/data.grb/catalog Waxman E., Bahcall J., 1997, Phys. Rev. Lett. 78, 2292; hep-ph/9807282 